\title{
Influence of COVID-19 pandemic on sexuality: a cross-sectional study among couples in Turkey
}

\author{
Mehmet Ali Karagöz $\mathbb{D}^{1} \cdot$ Abdullah Gül $^{2} \cdot$ Charmaine Borg $^{3} \cdot$ İsmet Bilger Erihan ${ }^{1} \cdot$ Mehmet Uslu $^{1} \cdot$ Mehmet Ezer $^{1}$ • \\ Ahmet Erbağcı ${ }^{1} \cdot$ Binali Çatak $^{4} \cdot$ Murat Bağcıoğlu ${ }^{1}$
}

Received: 22 June 2020 / Revised: 20 October 2020 / Accepted: 10 November 2020 / Published online: 16 December 2020

(c) The Author(s), under exclusive licence to Springer Nature Limited 2020

\begin{abstract}
Coronavirus disease 2019 (COVID-19) pandemic has been continuing to affect the lives of all people globally. It has been shown that restrictions due to changes in lifestyles lead to mental health problems. This study aims to investigate the effect of COVID-19 pandemic on couples' sexuality. A total of 245 volunteers (148 men and 97 women) were enrolled in the study. Generalized Anxiety Disorder-7, Patient Health Questionnaire, Perceived Stress Scale were administered to screen anxiety and depression symptoms. International Index of Erectile Function (IIEF-15) and Female Sexual Function Index (FSFI) along with self-constructed sexual behavior questionnaire were administered to participants, in order to evaluate sexual functions and behavioral changes during the pandemic. Sexual function scores (IIEF erectile function domain and total FSFI) during pandemic $(24.55 \pm 5.79$ and $24.87 \pm 7.88$, respectively) were lower compared to the prepandemic period (26.59 \pm 4.51 and $26.02 \pm 6.22$, respectively) $(p=0.001$ and $p=0.027$, respectively). During pandemic compared to prepandemic period, the frequency of sexual intercourse decreased in men $(p=0.001)$ and women $(p=0.001)$ while sexual avoidance and solitary sexual approach behaviors (masturbation or watching sexual content videos, etc.) increased in men $(p=0.001)$ and women $(p=0.022)$. However, the couples that spent more time together during the pandemic reported better sexual function scores (men; $p=0.001$, women; $p=0.006$ ). Although this is the first study evaluating couples from Turkey with a convenience sample, further studies with a greater number may better elucidate the effects of this pandemic on sexuality.
\end{abstract}

\section{Introduction}

Novel coronavirus (SARS-CoV-2) was detected firstly in December 2019 in China (Wuhan, Hubei) [1]. Then, it has rapidly spread all over the world and resulted in unpredictably changes in our lives. This novel coronavirus disease (COVID-19), which is transmitted by respiratory tract or by

Mehmet Ali Karagöz

dr_mali@msn.com

1 Department of Urology, Faculty of Medicine, Kafkas University, Kars, Turkey

2 Department of Urology, University of Health Sciences, Bursa Yuksek Ihtisas Training \& Research Hospital, Bursa, Turkey

3 Department of Clinical Psychology \& Experimental Psychopathology, University of Groningen, Groningen, Netherlands

4 Department of Public Health, Faculty of Medicine, Kafkas University, Kars, Turkey direct contact with infected surfaces, was declared as a pandemic by the World Health Organization (WHO) in March 2020 and triggered all countries to take extraordinary measures [2]. Almost all organizations (scientific, sportive, recreational, etc.) have been canceled and travel has been restricted. Quarantines and curfews have become daily routines and the concept of social distancing has become our new normality, even within families, causing significant disruptions in many social relationships.

Due to the fear of potential infection risk with COVID19, time spent at home has increased and restrictions on socializing and economic losses have caused anxiety and depression in many people who have been forced to stay at home during this period $[3,4]$. As it is known, quality and satisfying sexual life have a positive effect on the social and daily relationships as well as on the intimate life of many individuals $[5,6]$. The WHO summarizes the definition of sexual health as the physical, emotional, mental, and social well-being of an individual [7]. On the other hand, sexual dysfunction can be described as any condition preventing the individual from being satisfied by the sexual activity at 
any stage of the sexual relationship. In this manner, consistent evidence suggests that in addition to organic causes (vascular, hormonal, neurogenic, pharmacological) of sexual dysfunction, psychogenic causes, such as anxiety and depression also have a negative effect on sexual life, in both men and women $[8,9]$.

In a study conducted by Dunn et al, erectile dysfunction was observed 1.3-2.3-fold more in individuals with anxiety and depression [10]. Similarly, Mitchell et al., reported that females with depression had 3.12-fold sexual dysfunction [11]. Although the mechanism of the relationship between sexual dysfunction and psychological problems is not understood yet, it is known to be reciprocal and multifactorial [12]. The addition of sexual dysfunction can worsen the already present psychopathology and create a vicious circle [13-15]. Moreover, in a study of Hedon on male sexual dysfunction, it was emphasized that this vicious circle also impacted negatively on the partner and consequently on the relationship [9].

In this study, we aimed to evaluate the changes in the sexual lives of married or co-habiting couples in a Turkish sample during COVID-19 pandemic period which has been on-going for a long time.

\section{Methods and design}

\section{Population}

This cross-sectional study was approved by the NonInterventional Ethics Committee of Kafkas University, Faculty of Medicine (IRB No.: 80576354-050-99/187). This study consisted of 270 volunteers (162 men and 108 women). All the volunteers were sexually active before the pandemic period. Data were recorded from face-to-face interviews or via online questionnaire completion (Google Forms) between 6th of May 2020 and 20th of May 2020. Participants who were enrolled in the study by filling online questionnaires were reached via social media platform shares. Respondents were provided with the opportunity to check, review and change their answers. The rest of the participants who participated in face-to-face interviews were from volunteers who applied for outpatient treatment services of the hospital. After the aims, stages, and confidentiality of the study were explained to the participants, written or online informed consent was obtained from all volunteers. The data of the participants who were able to complete the form were transferred to the online record system. For various reasons, a total of 25 volunteers were excluded from the study (e.g., they were living separately from their partner during the pandemic, filled the questionnaire incompletely or were using antidepressant drugs). Thus, the final data consisted of 148 men and 97 women.

\section{Patient selection}

Criteria for inclusion in the study were defined as age 18-65 years and married or co-habiting hetereosexual couples who were living together before the pandemic and continued to do so during this period. Exclusion criteria were defined as men treated for erectile dysfunction, women with any sexual dysfunction hindering sexual relations before or during the pandemic, individuals and their partners treated for or suspected COVID-19 or isolated after testing, pregnant women and their partners and those previously under treatment for anxiety or depression. In the inclusion criteria, the participation of couples together was not compulsory in the study.

\section{Procedure}

In the first part of the study, along with demographic features, the Generalized Anxiety Disorder-7 (GAD-7) [16], Patient Health Questionnaire-9 (PHQ-9) [17], and Perceived Stress Scale (PSS) [18] were questioned to screen for anxiety and depression symptoms, all of which have been validated in Turkish language and have been shown to be valid and reliable. In the second part, general sexual tendencies and approaches during the pandemic period were evaluated in comparison with the period before the pandemic via a questionnaire constructed by us for the purpose of this study. Then, in the last part, to evaluate sexual functions, male participants completed the International Index of Erectile Function-15 (IIEF-15) questionnaire [19] and female participants completed the Female Sexual Function Index (FSFI) questionnaire [20]. The participants were instructed to complete the sexual function scales twice; in the first one, we offered them to complete the form based on their past experiences before the pandemic and in the second completion of the IIEF and FSFI these were done based on their experiences during the pandemic.

\section{Measures}

\section{Self-reports}

GAD-7 is accepted as a reliable tool, which is used to determine the severity of anxiety symptoms. Turkish validation of the GAD-7 was made by Konkan et al. in 2013 and consists of seven items according to DSM-IV criteria with 4-point Likert-type responses [21]. The PHQ-9 is a valid and reliable scale consisting of nine items with four Likert-type responses encompassing the DSM-IV criteria for the screening of depression. Turkish validation and reliability studies were conducted by Sari et al. in 2016 [22]. The PSS is a scale with proven validity and reliability, 
which measures the subjective stress perception of an individual with 14 items with 5-point Likert-type responses. Validity and reliability studies of the scale in Turkish were conducted by Eskin et al. in 2013 [23]. The IIEF-15 questionnaire is the most widely used scale in the evaluation of sexual desire, orgasm, intercourse satisfaction, and overall satisfaction in addition to male erectile function [17]. IIEF15 was translated and validated into Turkish and has been used in many studies [24-26]. The FSFI is a selfadministered scale that evaluates six components of female sexual function (sexual desire, arousal, lubrication, orgasm, satisfaction, and pain) in the previous 4 weeks with 19 items. Turkish validation of the scale was conducted by Aygin et al. in 2005 [27].

\section{Data analysis}

After the collection of all data, the participants were divided into two groups according to their sex. The demographic data and psychological status of the participants were evaluated in both groups. Changes in sexual behavior before and during the pandemic period of male and female participants were analyzed in respect of sexes and within sexes. Then in both males and females, sexual function scores (IIEF and FSFI) and all domains during the pandemic were compared with the period before the pandemic. Finally, the evaluation of correlation analysis of sexual function scores was done by using GAD-7, PHQ-9, PSS, age, and economic loss.

According to the result of power analysis (using G*Power 3.1 program) with a 0.90 power value, a 0.05 error and a 0.3 effect size, 97 participants for a type of sex were enough for evaluation. Data obtained in the study were analyzed using SPSS software version 24.0. The normality of distribution of continuous variables was tested with Shapiro-Wilk test. Whereas independent $t$ test and Mann-Whitney $U$ test were used for comparison of two independent groups, dependent groups were analyzed by Wilcoxon test. The Chi-square test was used to assess the relationships between categorical variables and Spearman rank correlation coefficients were calculated to show correlations between the variables. A value of $p<0.05$ was accepted as statistically significant.

\section{Results}

The demographic data and results of psychological status scales of the study population, which consists of married and co-habiting couples, are presented in Table 1. Female participants were younger than males $(p=0.032)$. The pandemic had a negatively economic effect (small, moderate, large) on $49.3 \%$ of the males and on $48.5 \%$ of the females $(p=0.359)$.
According to the GAD-7, anxiety symptoms in male participants were found to be mild in $52 \%$, moderate in $8.8 \%$, and severe in $2 \%$. In the female subjects, anxiety symptoms were observed to be mild in $59.8 \%$, moderate in $19.6 \%$, and severe in $5.2 \%$. It was significantly higher in female volunteers $(p=0.001)$. According to the results of the PHQ-9 used in the screening for depression, the depression symptoms were determined as mild in $56.8 \%$, moderate in $10.1 \%$, and moderate to severe in $6.8 \%$ of the males, and mild in $51.5 \%$, moderate in $24.7 \%$, moderate to severe in $11.3 \%$, and severe in $2.1 \%$ of the females. Severity of the depression symptoms was also significantly higher in females $(p=0.001)$. The mean PSS points were determined as $22.16 \pm 6.84$ for males and $26.95 \pm 6.8$ for females. The difference was found to be significantly higher in females $(p=0.001)$.

During the pandemic, $75.7 \%$ of the male and $76.3 \%$ of the female respondents reported spending more time with their partner $(p=0.913)$ (Table 2). The restrictions and limitations during this period were perceived as positive in the daily and emotional relationship with their partner, in $31.8 \%$ of males and in $32 \%$ of females $(p=0.91)$. Behavior to avoid sexual closeness with the partner due to concern of spreading COVID-19 was reported by $19.6 \%$ of males and $38.1 \%$ of females $(p=0.001)$. Thoughts that participants could be infected during sexual intercourse were expressed by $15.5 \%$ of males and by $32 \%$ of females $(p=0.002)$. Precautions like using condoms or avoidance from kissing were taken by $6.8 \%$ of males and $14 \%$ of females $(p=0.048)$. An increase in solitary sexual activity (masturbation or watching sexual content videos, etc.) during this period was reported by $12.8 \%$ of males and $4.1 \%$ of females $(p=0.022)$. This approach had not been used previously but was adopted during the pandemic by $3.4 \%$ of males and $5.25 \%$ of females $(p=0.492)$. The frequency of sexual intercourse during the pandemic reduced significantly in both sexes compared to the period before the pandemic $(p=0.001)$ (Table 3).

When compared, the period of the pandemic with the preceding period in terms of IIEF domains of men, a significant decrease was determined in the erectile and orgasmic function, intercourse satisfaction, and overall satisfaction scores $(p=0.001, p=0.014, p=0.001, p=$ 0.001 , respectively) and no significant decrease was found in the sexual desire domain score $(p=0.173)$ (Table 4). During pandemic compared to prepandemic period, the total FSFI score decreased from 26.02 to $24.87(p=0.027)$ (Table 5). The decrease that was determined in the FSFI desire, arousal, and pain domains did not reach to a significant statistical difference $(p=0.646, p=0.103, p=$ 0.065 , respectively). A statistically significant decrease was determined in the domains of lubrication, orgasm, and satisfaction in women $(p=0.034, p=0.023, p=0.007$, respectively). 
Table 1 Demographics and psychological status of the participants.

\begin{tabular}{|c|c|c|c|c|}
\hline Variables & $\begin{array}{l}\text { Total } \\
(n=245)\end{array}$ & $\begin{array}{l}\text { Female } \\
(n=97)\end{array}$ & $\begin{array}{l}\text { Male } \\
(n=148)\end{array}$ & $\begin{array}{l}\text { Female vs. male } \\
p \text { value }\end{array}$ \\
\hline Age, year $($ mean $\pm S D)$ & $35.9 \pm 6.9$ & $34.7 \pm 6.67$ & $36.7 \pm 7.1$ & $0.032^{\mathrm{a}}$ \\
\hline Height, cm $($ mean $\pm \mathrm{SD})$ & $172.7 \pm 8.7$ & $164.5 \pm 5.65$ & $178.1 \pm 5.7$ & $0.001^{\mathrm{a}}$ \\
\hline Weight, $\mathrm{kg}($ mean $\pm \mathrm{SD})$ & $76.9 \pm 15.4$ & $65.8 \pm 12.41$ & $84.2 \pm 12.7$ & $0.001^{\mathrm{a}}$ \\
\hline BMI, $\mathrm{kg} / \mathrm{m}^{2}($ mean $\pm \mathrm{SD})$ & $25.61 \pm 3.94$ & $24.25 \pm 4.15$ & $26.51 \pm 3.54$ & $0.001^{\mathrm{a}}$ \\
\hline Smoker, $n(\%)$ & $95(38.8)$ & $25(25.8)$ & $70(47.3)$ & $0.001^{\mathrm{a}}$ \\
\hline Alcohol consumer, $n(\%)$ & $85(34.7)$ & $23(23.7)$ & $62(41.9)$ & $0.003^{\mathrm{a}}$ \\
\hline Regular exercise, $n(\%)$ & $74(30.2)$ & $23(23.7)$ & $51(34.5)$ & 0.073 \\
\hline Education level, $n(\%)$ & & & & $0.001^{\mathrm{a}}$ \\
\hline Primary school & $2(0.8)$ & $0(0)$ & $2(1.4)$ & \\
\hline High school & $32(13.1)$ & $9(9.3)$ & $23(15.5)$ & \\
\hline University & $136(55.5)$ & $65(67)$ & $71(48)$ & \\
\hline Post graduate/PhD & $75(30.6)$ & $23(23.7)$ & $52(35.1)$ & \\
\hline Comorbidities, $n(\%)$ & $38(15.5)$ & $21(21.6)$ & 17 (11.5) & $0.032^{\mathrm{a}}$ \\
\hline Hypertension & $11(4.5)$ & $2(2.1)$ & $9(6.1)$ & 0.137 \\
\hline Diabetes mellitus & $12(4.9)$ & $3(3.1)$ & $9(6.1)$ & 0.289 \\
\hline Hyperlipidemia & $3(1.2)$ & $2(2.1)$ & $1(0.7)$ & 0.564 \\
\hline Coronary artery disease & $1(0.4)$ & $0(0)$ & $1(0.7)$ & 0.417 \\
\hline Other & $20(8.2)$ & $16(16.5)$ & $4(2.7)$ & $0.001^{\mathrm{a}}$ \\
\hline Medication usage, $n(\%)$ & $40(16.3)$ & $28(28.9)$ & $12(8.1)$ & $0.001^{\mathrm{a}}$ \\
\hline Duration of marriage/partnership, $n(\%)$ & & & & 0.233 \\
\hline$\leq 1$ year & $25(10.2)$ & $7(7.2)$ & $18(12.2)$ & \\
\hline $1-5$ years & $74(30.2)$ & $32(33)$ & $42(28.4)$ & \\
\hline $5-10$ years & $67(27.3)$ & $22(22.7)$ & $45(30.4)$ & \\
\hline$\geq 10$ years & $79(32.2)$ & $36(37.1)$ & $43(29.1)$ & \\
\hline Negative effect of pandemic on economic status, $n(\%)$ & & & & 0.359 \\
\hline None & $125(51)$ & $50(51.5)$ & $75(50.7)$ & \\
\hline Mild & $57(23.3)$ & $22(22.7)$ & $35(23.6)$ & \\
\hline Moderate & 48 (19.6) & $22(22.7)$ & $26(17.6)$ & \\
\hline Severe & $15(6.1)$ & $3(1.2)$ & $12(8.1)$ & \\
\hline GAD-7 score, $n(\%)$ & & & & $0.001^{\mathrm{a}}$ \\
\hline No anxiety & $70(28.6)$ & $15(15.5)$ & $55(37.2)$ & \\
\hline Mild anxiety & $135(55.1)$ & $58(59.8)$ & $77(52)$ & \\
\hline Moderate anxiety & $32(13.1)$ & 19 (19.6) & $13(8.8)$ & \\
\hline Severe anxiety & $8(3.3)$ & $5(5.2)$ & $3(2)$ & \\
\hline PHQ-9 score, $n(\%)$ & & & & $0.001^{\mathrm{a}}$ \\
\hline No depression & $49(20)$ & $10(10.3)$ & $39(26.4)$ & \\
\hline Mild depression & $134(54.7)$ & $50(51.5)$ & $84(56.8)$ & \\
\hline Moderate depression & $39(15.9)$ & $24(24.7)$ & $15(10.1)$ & \\
\hline Moderate to severe depression & $21(8.6)$ & $11(11.3)$ & $10(6.8)$ & \\
\hline Severe depression & $2(0.8)$ & $2(2.1)$ & $0(0)$ & \\
\hline PSS score $($ mean $\pm \mathrm{SD})$ & $24.06 \pm 7.20$ & $26.95 \pm 6.80$ & $22.16 \pm 6.84$ & $0.001^{\mathrm{a}}$ \\
\hline GAD-7 score $($ mean $\pm \mathrm{SD})$ & $6.49 \pm 3.64$ & $7.56 \pm 3.74$ & $5.8 \pm 3.4$ & $0.001^{\mathrm{a}}$ \\
\hline PHQ-9 score $($ mean \pm SD) & $7.79 \pm 4.32$ & $9.17 \pm 4.59$ & $6.89 \pm 3.9$ & $0.001^{\mathrm{a}}$ \\
\hline
\end{tabular}

GAD-7 Generalized Anxiety Disorder Score, PHQ-9 score Patient Health Questionnaire score, PSS Perceived Stress Scale score.

a'Significant at 0.05 level. Student's $t$ test and Mann-Whitney $U$ test for numerical, Chi-square test for categorical data. 
Table 2 Changes of sexual behaviors during COVID-19 pandemic.

\begin{tabular}{|c|c|c|c|c|}
\hline Variables & Total $(n=245)$ & Female $(n=97)$ & Male $(n=148)$ & $\begin{array}{l}\text { Female vs. male } \\
p \text { value }\end{array}$ \\
\hline Spending more time with partner, $n(\%)$ & $186(75.9)$ & $74(76.3)$ & $112(75.7)$ & 0.913 \\
\hline $\begin{array}{l}\text { Effect of restrictions and limitations on your daily and emotional } \\
\text { relationships, } n(\%)\end{array}$ & & & & 0.910 \\
\hline No difference & $122(49.8)$ & $47(48.5)$ & $75(50.7)$ & \\
\hline Positively & $78(31.8)$ & $31(32)$ & $47(31.8)$ & \\
\hline Negatively & $45(18.4)$ & $19(19.6)$ & $26(17.6)$ & \\
\hline Sexual avoidance behaviors towards your partner, $n(\%)$ & $66(26.9)$ & $37(38.1)$ & $29(19.6)$ & $0.001^{\mathrm{a}}$ \\
\hline Considering that you could be infected during intercourse, $n(\%)$ & $55(22.0)$ & $31(32.0)$ & $23(15.5)$ & $0.002^{\mathrm{a}}$ \\
\hline Considering that partner has sexual avoidance behaviors, $n(\%)$ & $38(15.5)$ & $15(15.5)$ & $23(15.5)$ & 0.987 \\
\hline Being insightful of partners sexual avoidance behaviors, $n(\%)$ & $128(52.2)$ & $49(50.5)$ & $79(53.4)$ & 0.883 \\
\hline Taking precautions during intercourse, $n(\%)$ & $24(9.8)$ & $14(14.4)$ & $10(6.8)$ & $0.048^{\mathrm{a}}$ \\
\hline $\begin{array}{l}\text { Increase in solitary sexual satisfaction approach (masturbation, } \\
\text { video etc), } n(\%)\end{array}$ & $23(9.4)$ & $4(4.1)$ & $19(12.8)$ & $0.022^{\mathrm{a}}$ \\
\hline New onset of solitary sexual satisfaction approach, $n(\%)$ & $10(4.1)$ & $5(5.2)$ & $5(3.4)$ & 0.492 \\
\hline
\end{tabular}

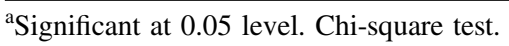

Table 3 Comparison of intercourse frequencies before and during COVID-19 pandemic.

\begin{tabular}{|c|c|c|c|c|c|c|}
\hline \multirow{2}{*}{$\begin{array}{l}\text { Frequency of } \\
\text { intercourse } \\
\text { per week }\end{array}$} & \multicolumn{3}{|l|}{ Female } & \multicolumn{3}{|l|}{ Male } \\
\hline & $\begin{array}{l}\text { Before } \\
\text { pandemic } \\
n(\%)\end{array}$ & $\begin{array}{l}\text { During } \\
\text { pandemic } \\
n(\%)\end{array}$ & $p$ value & $\begin{array}{l}\text { Before } \\
\text { pandemic } \\
n(\%)\end{array}$ & $\begin{array}{l}\text { During } \\
\text { pandemic } \\
n(\%)\end{array}$ & $p$ value \\
\hline & & & $0.001^{\mathrm{a}}$ & & & $0.001^{\mathrm{a}}$ \\
\hline$\leq 1$ & $17(17.5)$ & $40(41.2)$ & & $28(18.9)$ & $67(45.3)$ & \\
\hline 2 & $47(48.5)$ & $33(34)$ & & 66 (44.6) & $41(27.7)$ & \\
\hline $2-5$ & $29(29.9)$ & $21(21.6)$ & & $48(32.4)$ & $30(20.3)$ & \\
\hline$\geq 5$ & $4(4.1)$ & $3(3.1)$ & & $6(4.1)$ & $10(6.8)$ & \\
\hline
\end{tabular}

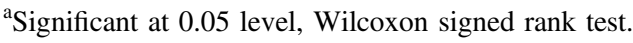

Table 4 Comparison of IIEF-15 scores before and during COVID-19 pandemic in men $(n=148)$.

\begin{tabular}{|c|c|c|c|}
\hline IIEF domains & $\begin{array}{l}\text { Before pandemic } \\
(\text { mean } \pm S D)\end{array}$ & $\begin{array}{l}\text { During pandemic } \\
(\text { mean } \pm S D)\end{array}$ & $p$ value \\
\hline Erectile function & $26.59 \pm 4.51$ & $24.55 \pm 5.79$ & $0.001^{\mathrm{a}}$ \\
\hline $\begin{array}{l}\text { Orgasmic } \\
\text { function }\end{array}$ & $9.11 \pm 1.37$ & $8.64 \pm 2.3$ & $0.014^{\mathrm{a}}$ \\
\hline Sexual desire & $7.75 \pm 1.49$ & $7.57 \pm 1.82$ & 0.173 \\
\hline $\begin{array}{l}\text { Intercourse } \\
\text { satisfaction }\end{array}$ & $11.53 \pm 2.32$ & $10.66 \pm 3.64$ & $0.001^{\mathrm{a}}$ \\
\hline $\begin{array}{l}\text { Overall } \\
\text { satisfaction }\end{array}$ & $8.58 \pm 1.66$ & $8.04 \pm 2.16$ & $0.001^{\mathrm{a}}$ \\
\hline
\end{tabular}

IIEF International Index of Erectile Function.

${ }^{\text {a }}$ Significant at 0.05 level, Wilcoxon test.

Erectile function domain had significantly negative correlation with economic loss ( $p=0.001 ; r=-0.328)$, GAD$7(p=0.001 ; r=-0.297)$, PHQ-9 $(p=0.006 ; r=-0.224)$, and PSS $(p=0.001 ; r=-0.346)$ (Table 6). There was
Table 5 Comparison of FSFI scores before and during COVID-19 pandemic in women $(n=97)$.

\begin{tabular}{llll}
\hline FSFI domains & $\begin{array}{l}\text { Before pandemic } \\
(\text { mean } \pm \text { SD) }\end{array}$ & $\begin{array}{l}\text { During pandemic } \\
(\text { mean } \pm \text { SD })\end{array}$ & $p$ value \\
\hline Total Score & $26.02 \pm 6.22$ & $24.87 \pm 7.88$ & $0.027^{\mathrm{a}}$ \\
Desire & $3.77 \pm 1.08$ & $3.74 \pm 1.33$ & 0.646 \\
Arousal & $4.01 \pm 1.19$ & $3.80 \pm 1.51$ & 0.103 \\
Lubrication & $4.77 \pm 1.15$ & $4.55 \pm 1.48$ & $0.034^{\mathrm{a}}$ \\
Orgasm & $4.25 \pm 1.43$ & $4.04 \pm 1.61$ & $0.023^{\mathrm{a}}$ \\
Satisfaction & $4.41 \pm 1.35$ & $4.11 \pm 1.53$ & $0.007^{\mathrm{a}}$ \\
Pain & $4.78 \pm 1.42$ & $4.60 \pm 1.58$ & 0.065 \\
FSFI< & 45.4 & 52.6 & $0.001^{\mathrm{a}}$ \\
26.55 $(\%)$ & & & \\
\hline
\end{tabular}

FSFI Female Sexual Function Index.

${ }^{\text {a }}$ Significant at 0.05 level, Wilcoxon test, and Chi-square test.

no significant correlation between age and erectile function $(p=0.528 ; r=-0.052)$. The males who reported spending more time with their partner during the pandemic, reported 
Table 6 Correlations of IIEF-15 domains with age, economic loss, GAD-7, PHQ-9, and PSS.

\begin{tabular}{|c|c|c|c|c|c|}
\hline Correlation factors & $\begin{array}{l}\text { Erectile } \\
\text { function }\end{array}$ & $\begin{array}{l}\text { Orgasmic } \\
\text { function }\end{array}$ & Sexual desire & $\begin{array}{l}\text { Intercourse } \\
\text { satisfaction }\end{array}$ & $\begin{array}{l}\text { Overall } \\
\text { satisfaction }\end{array}$ \\
\hline Age & $\begin{array}{l}r=-0.052 \\
p=0.528\end{array}$ & $\begin{array}{l}r=0.064 \\
p=0.442\end{array}$ & $\begin{array}{l}r=-0.041 \\
p=0.622\end{array}$ & $\begin{array}{l}r=-0.096 \\
p=0.248\end{array}$ & $\begin{array}{l}r=-0.049 \\
p=0.557\end{array}$ \\
\hline Economic loss & $\begin{array}{l}r=-0.328 \\
p=0.001^{\mathrm{a}}\end{array}$ & $\begin{array}{l}r=-0.098 \\
p=0.234\end{array}$ & $\begin{array}{l}r=-0.092 \\
p=0.268\end{array}$ & $\begin{array}{l}r=-0.210 \\
p=0.011^{\mathrm{a}}\end{array}$ & $\begin{array}{l}r=-0.104 ; \\
p=0.210\end{array}$ \\
\hline GAD-7 score $(\%)$ & $\begin{array}{l}r=-0.297 \\
p=0.001^{\mathrm{a}}\end{array}$ & $\begin{array}{l}r=-0.104 \\
p=0.210\end{array}$ & $\begin{array}{l}r=-0.199 \\
p=0.015^{\mathrm{a}}\end{array}$ & $\begin{array}{l}r=-0.202 \\
p=0.014^{\mathrm{a}}\end{array}$ & $\begin{array}{l}r=-0.219 ; \\
p=0.007^{\mathrm{a}}\end{array}$ \\
\hline PHQ-9 score (\%) & $\begin{array}{l}r=-0.224 \\
p=0.006^{\mathrm{a}}\end{array}$ & $\begin{array}{l}r=-0.089 \\
p=0.281\end{array}$ & $\begin{array}{l}r=-0.149 \\
p=0.071\end{array}$ & $\begin{array}{l}r=-0.215 \\
p=0.009^{\mathrm{a}}\end{array}$ & $\begin{array}{l}r=-0.175 \\
p=0.033^{\mathrm{a}}\end{array}$ \\
\hline PSS score & $\begin{array}{l}r=-0.346 \\
p=0.001^{\mathrm{a}}\end{array}$ & $\begin{array}{l}r=-0.133 \\
p=0.107\end{array}$ & $\begin{array}{l}r=-0.106 \\
p=0.200\end{array}$ & $\begin{array}{l}r=-0.330 \\
p=0.001^{\mathrm{a}}\end{array}$ & $\begin{array}{l}r=-0.179 ; \\
p=0.030^{\mathrm{a}}\end{array}$ \\
\hline
\end{tabular}

IIEF International Index of Erectile Function, GAD-7 Generalized Anxiety Disorder Score, $P H Q-9$ Score Patient Health Questionnaire Score, $P S S$ Perceived Stress Scale Score.

${ }^{\text {a }}$ Significant at 0.05 level, Spearman rank correlation coefficient.

Table 7 Correlations of total FSFI score and FSFI domains with age, economic loss, GAD-7, PHQ-9, and PSS.

\begin{tabular}{|c|c|c|c|c|c|c|c|}
\hline Correlation factors & FSFI desire & FSFI arousal & FSFI lubrication & FSFI orgasm & FSFI satisfaction & FSFI pain & FSFI total \\
\hline Age & $\begin{array}{l}r=-0.195 \\
p=0.056\end{array}$ & $\begin{array}{l}r=-0.214 \\
p=0.036^{\mathrm{a}}\end{array}$ & $\begin{array}{l}r=-0.228 \\
p=0.025^{\mathrm{a}}\end{array}$ & $\begin{array}{l}r=-0.134 \\
p=0.190\end{array}$ & $\begin{array}{l}r=-0.204 \\
p=0.045^{\mathrm{a}}\end{array}$ & $\begin{array}{l}r=-0.229 \\
p=0.024^{\mathrm{a}}\end{array}$ & $\begin{array}{l}r=-0.238 \\
p=0.019^{\mathrm{a}}\end{array}$ \\
\hline Economic loss & $\begin{array}{l}r=-0.161 \\
p=0.114\end{array}$ & $\begin{array}{l}r=-0.071 \\
p=0.490\end{array}$ & $\begin{array}{l}r=-0.067 \\
p=0.516\end{array}$ & $\begin{array}{l}r=-0.040 \\
p=0.697\end{array}$ & $\begin{array}{l}r=-0.033 \\
p=0.749\end{array}$ & $\begin{array}{l}r=-0.109 \\
p=0.287\end{array}$ & $\begin{array}{l}r=-0.089 ; \\
p=0.385\end{array}$ \\
\hline GAD-7 score & $\begin{array}{l}r=-0.263 \\
p=0.009^{\mathrm{a}}\end{array}$ & $\begin{array}{l}r=-0.254 \\
p=0.012^{\mathrm{a}}\end{array}$ & $\begin{array}{l}r=-0.275 \\
p=0.006^{\mathrm{a}}\end{array}$ & $\begin{array}{l}r=-0.298 \\
p=0.003^{\mathrm{a}}\end{array}$ & $\begin{array}{l}r=-0.314 \\
p=0.002^{\mathrm{a}}\end{array}$ & $\begin{array}{l}r=-0.144 \\
p=0.160\end{array}$ & $\begin{array}{l}r=-0.317 \\
p=0.002^{\mathrm{a}}\end{array}$ \\
\hline PHQ-9 score & $\begin{array}{l}r=-0.293 \\
p=0.004^{\mathrm{a}}\end{array}$ & $\begin{array}{l}r=-0.239 \\
p=0.019^{\mathrm{a}}\end{array}$ & $\begin{array}{l}r=-0.197 \\
p=0.053\end{array}$ & $\begin{array}{l}r=-0.233 \\
p=0.022^{\mathrm{a}}\end{array}$ & $\begin{array}{l}r=-0.279 \\
p=0.006^{\mathrm{a}}\end{array}$ & $\begin{array}{l}r=-0.056 \\
p=0.586\end{array}$ & $\begin{array}{l}r=-0.268 \\
p=0.008^{\mathrm{a}}\end{array}$ \\
\hline PSS score & $\begin{array}{l}r=-0.392 \\
p=0.001^{\mathrm{a}}\end{array}$ & $\begin{array}{l}r=-0.271 \\
p=0.007^{\mathrm{a}}\end{array}$ & $\begin{array}{l}r=-0.158 \\
p=0.123\end{array}$ & $\begin{array}{l}r=-0.260 \\
p=0.010^{\mathrm{a}}\end{array}$ & $\begin{array}{l}r=-0.295 \\
p=0.003^{\mathrm{a}}\end{array}$ & $\begin{array}{l}r=-0.096 \\
p=0.350\end{array}$ & $\begin{array}{l}r=-0.290 \\
p=0.004^{\mathrm{a}}\end{array}$ \\
\hline
\end{tabular}

FSFI Female Sexual Function Index, GAD-7 Generalized Anxiety Disorder Score, PHQ-9 Score Patient Health Questionnaire Score, PSS Perceived Stress Scale Score.

${ }^{a}$ Significant at 0.05 level, Spearman rank correlation coefficient.

higher scores in all IIEF domains compared to those who reported that they were not spending more time with their partner (erectile function; $p=0.001$, orgasmic function; $p=0.001$, sexual desire; $p=0.009$, intercourse satisfaction; $p=0.001$, overall satisfaction; $p=0.001)$.

Correlation analysis was applied to test any relationship between FSFI domains and age, economic loss, GAD-7, PHQ-9, and PSS (Table 7). No statistically significant correlation was determined between the decrease in total FSFI score during the pandemic and economic loss $(r=-0.089 ; p=0.38)$. Total FSFI scores had significantly negative correlation with age $(r=0.238 ; p=$ 0.019), GAD-7 ( $r=-0.317 ; p=0.002)$, PHQ-9 $\quad(r=$ $-0.268 ; p=0.008)$, PSS $(r=-0.290 ; p=0.004)$. The total FSFI and other domain scores for females who spent more time with their partner were higher than for those who did not spend more time with their partner (desire; $p=0.021$, arousal; $p=0.009$, lubrication; $p=0.01$, orgasm; $p=0.01$, satisfaction; $p=0.004$, pain; $p=0.046$, FSFI total; $p=0.006$ ).

\section{Discussion}

In this study, we aimed to examine the effects of the global COVID-19 pandemic on the sexual lives of couples in Turkey. According to the data obtained from the study, as the severity of anxiety, depression, and stress perception increased in this period, an increase in sexual dysfunctions was observed in both male and female volunteers. These findings were found to be consistent with those of Yang et al. and Nicolosi et al. [28, 29].

In a study of Gillespie, it was stated that the frequency of sexual intercourse is one of the main factors that determine the individuals' sexual satisfaction status [30]. In another recent study conducted by Fernandes et al., sexual satisfaction was found to be highest in the group that had sexual intercourse 4-6 times per week [31]. Consistent with these reports, the frequency of sexual intercourse and overall sexual satisfaction levels decreased in both sexes of the current study population during pandemic compared to prepandemic period $(p=0.001)$. On the other hand, the 
increase in the frequency of sexual intercourse reported by $8.7 \%$ of males and $11.3 \%$ of females in the current study can be explained with the increase in the time spent together with their partner. In contrast to our results, in a recent study conducted in Italy during COVID-19 pandemic, reduction in sexual intercourse was not recorded [32].

In several studies to date, sexual avoidance behaviors have generally been associated with different conditions, such as psychiatric diseases (major depression, obsessive compulsive disorder, etc.) [33, 34], problems between the couples (lack of intimacy, attachment problems) [35-37] or chronic diseases (hypertension, coronary artery disease, cancer, rheumatoid arthritis, epilepsy, migraine) [38-42], among other reasons. As the incubation period of COVID19 can last for up to 2 weeks and individuals can be asymptomatic during that time, it may lead to concerns that the infection could be transmitted during sexual intercourse [43]. In the current study, it was seen that females had twofold higher sexual avoidance behaviors than males in this period $(p=0.001)$. It can be explained by the fact that the perception of stress in women is significantly higher than that of men $(p=0.001)$. Although it is not yet fully known whether the virus can be passed via semen or vaginal secretions in addition to the respiratory tract and direct contact [44-46], precautions, such as willingness to use a condom during intercourse and avoiding kissing were preferred twofold higher by females than by males $(p=$ 0.048). Sexual activity with a partner is known to provide a higher rate of sexual satisfaction than solitary sexual activity [47]. However, during the pandemic there was an increase in solitary sexual satisfaction approaches in both sexes, and the rate of this increase was significantly higher in males $(p=0.022)$ than in female participants. In accordance with the results of the current study, previous studies have reported that males are more predisposed than females to start solitary or dyadic sexual activity [48, 49]. Besides, as sexuality is still regarded as taboo in some sections of the Turkish society, this can contribute in explaining the difference in the rates of solitary sexual satisfaction approaches of the males and females in this study.

In the current study, the IIEF-15 scores during the pandemic were significantly lower compared to the period before the pandemic $(p=0.001)$. Low rates of severe erectile function disorder in both periods, and the relatively high IIEF scores could be attributed to excluding subjects who had been or were being treated for erectile dysfunction, the low rate of chronic diseases and that the mean age of the sample was young. Thus, it was aimed to clarify the effects of the pandemic period on the sexual lives with excluding other confounding reasons. Although there are some conflicting reports, in most studies it has been reported that the severity of anxiety and depression increases the loss of sexual desire $[14,50]$. Although a decrease in all the IIEF domain scores was observed, only the difference in the sexual desire domain was not statistically significant $(p=$ $0.17)$. The scores of the couples who spent more time together during the pandemic were determined to be significantly higher in all the domains of the IIEF scale, than those of the couples who did not spend more time together. Having more time together during the pandemic might have been helpful in balancing the negative effects of the pandemic.

The total FSFI scores of the females, who had more anxiety and depression symptoms and stress perception than males in the pandemic period, were significantly lower during the pandemic than in the preceding period $(p=$ 0.027). A decrease was observed in all the domains of the FSFI score, but the differences in sexual desire, arousal, and pain domains were not statistically significant. As stated above, this could be explained by an increase in closeness (emotional intimacy, bonding) and positive changes in the relationship with the spouse or partner reported by more than $30 \%$ of both sexes during the pandemic. This explanation is compatible with Basson's sexual response model, which is based on the closeness, intimacy, bonding, and compatibility established with the partner beyond sexual need and desire [51]. Nevertheless, we would like to emphasize that the presence of multiple factors that may have an impact on both the IIEF and FSFI scores during the pandemic may have affected the strength of the correlation analysis.

In previous studies, it has been demonstrated that events that cause trauma at a societal level have an impact on the sexual health as well as on the psychological well-being of many individuals. In a study investigating the effect of earthquake that occurred in Wenchuan, on female sexual behaviors, a significant decrease in sexual satisfaction was reported [52]. Similarly, Kissinger reported differences in sexual behaviors following hurricane Katrina in the USA [53]. In this context, new studies have also been conducted about the COVID-19 pandemic. It was stated in a crossnational study conducted by Arafat et al. that the rate of the individuals with a sexual intercourse frequency between one and five per week before the pandemic decreased from 76.7 to $72.5 \%$ during pandemic whereas the rate of those with an intercourse frequency more than five per week increased by 3.3\% [54]. A study from Turkey revealed that a significant increase in the frequency of sexual intercourse was shown in the pandemic period compared to prepandemic one while the total FSFI score decreased significantly [55]. In another Italy-based study conducted on reproductive-age women, it was shown that both the FSFI score and the frequency of sexual intercourse decreased during pandemic [56]. It was found in an online survey study including 3500 individuals 
that the frequency of sexual intercourse decreased in $41 \%$ of the participants, and frequency of masturbation increased in $30 \%$ of those [57]. Lastly, in a recent study from China conducted in the period of COVID-19, it was shown that total sexual satisfaction and frequency ratio decreased, which is in accordance with the current study [58].

Although this is the first study evaluating married or co-habiting couples in terms of different sexual perspectives during the COVID-19 pandemic in Turkey, there are some limitations to be considered. Firstly, in societies like Turkey (in which sexuality may still be considered as a taboo), especially face-to-face interviews might have an impact on the answers about sexuality. The participants were asked to compare intercourse frequency and the FSFI and IIEF forms with the period before the pandemic. The data collection via questionnaire from past experiences may differ according to the individual's motivation and psychology at that time, and can cause self-reporting biases. Moreover, the living conditions (children staying at home due to closed school, small house, etc.) were not questioned that might also influence intimate life of couples. Although the number of subjects included was sufficient, the sample may not represent all segments of society, so one has to be cautious before generalizing these findings. Further studies with greater numbers of subjects would be able to provide more reliable results. Moreover, as the study was planned to be cross-sectional, no causal inferences can be made.

In conclusion, COVID-19 pandemic is a period in which many different factors can have a positive or negative effect on sexual behaviors. From the results of this study, a significant decrease was observed in the frequency of sexual intercourse and general satisfaction in pandemic period. In addition, a significant increase in sexual avoidance behavior of females and an increase in solitary sexual satisfaction approaches of males were observed. Further studies from different countries and cultures are needed to clarify the effect of this unprecedented pandemic and its consequences on sexual satisfaction and experiences. It is not known how long the pandemic will continue and scientific authorities warn to be cautious about the peak of new cases. Therefore, prolonging of this period may create an important duty for healthcare providers in respect of maintaining sexual health in addition to the physical and mental health of individuals

\section{Compliance with ethical standards}

Conflict of interest The authors declare that they have no conflict of interest.

Publisher's note Springer Nature remains neutral with regard to jurisdictional claims in published maps and institutional affiliations.

\section{References}

1. Zhu N, Zhang DY, Wang WL, Li X, Yang B, Song J. A novel coronavirus from patients with pneumonia in China. N Engl Med. 2019;382:727-33.

2. World Health Organization. Naming the coronavirus disease (COVID-19) and the virus that causes it. World Health Organization; 2020. https://www.who.int/emergencies/diseases/novelcoronavirus-2019/technical-guidance/naming-the-coronavirusdisease-(covid-2019)-and-the-virus-that-causes-it.

3. Torales J, O'Higgins M, Castaldelli-Maia JM, Ventriglio A. The outbreak of COVID-19 coronavirus and its impact on global mental health. Int J Soc Psychiatry. 2020;66:317-20.

4. Özdin S, Bayrak Özdin Ş. Levels and predictors of anxiety, depression and health anxiety during COVID-19 pandemic in Turkish society: the importance of gender. Int J Soc Psychiatry. 2020. https://doi.org/10.1177/0020764020927051.

5. Van Lankveld J, Jacobs N, Thewissen V, Dewitte M, Verboon P. The associations of intimacy and sexuality in daily life: temporal dynamics and gender effects within romantic relationships. J Soc Pers Relat. 2018;35:557-76.

6. Flynn KE, Lin L, Bruner DW, Cyranowski JM, Hahn EA, Jeffery $\mathrm{DD}$, et al. Sexual satisfaction and the importance of sexual health to quality of life throughout the life course of US adults. J Sex Med. 2016;13:1642-50.

7. World Health Organization. Sexual and reproductive health: defining sexual health. WHO; 2006. https://www.who.int/ reproductivehealth/topics/sexual_health/sh_definitions/en/.

8. Clayton AH, Groth J. Etiology of female sexual dysfunction. Women's Health. 2013;9:135-7.

9. Hedon F. Anxiety and erectile dysfunction: a global approach to ED enhances results and quality of life. Int J Impot Res. 2003;15: S16-9.

10. Dunn KM, Croft PR, Hackett GI. Association of sexual problems with social, psychological, and physical problems in men and women: a cross sectional population survey. J Epidemiol Community Health. 1999;53:144-8.

11. Mitchell KR, Mercer CH, Ploubidis GB, Jones KG, Datta J, Field $\mathrm{N}$, et al. Sexual function in Britain: findings from the third national survey of sexual attitudes and lifestyles (Natsal-3). Lancet. 2013;26:1-13.

12. Forbes MK, Baillie AJ, Schniering CA. A structural equation modeling analysis of the relationships between depression, anxiety, and sexual problems over time. J Sex Res. 2016;53:942-54.

13. Michael A, O'Keane V. Sexual dysfunction in depression. Hum Psychopharmacol Clin Exp 2000;15:337-45.

14. Minnen AV, Kampman M. The interaction between anxiety and sexual functioning: a controlled study of sexual functioning in women with anxiety disorders. Sex Relatsh Ther. 2000;15:47-57.

15. Bradford A, Meston CM. The impact of anxiety on sexual arousal in women. Behav Res Ther. 2006;44:1067-77.

16. Spitzer RL, Kroenke K, Williams JB, Löwe B. A brief measure for assessing generalized anxiety disorder: the GAD-7. Arch Intern Med. 2006;166:1092-7.

17. Kroenke K, Spitzer RL, Williams JB. The PHQ-9: validity of a brief depression severity measure. J Gen Intern Med. 2001;16: 606-13.

18. Cohen S, Kamarck T, Mermelstein R. A global measure of perceived stress. J Health Soc Behav. 1983;24:385-96.

19. Rosen RC, Riley A, Wagner G, Osterloh IH, Kirkpatrick J, Mishra A. Urology the international index of erectile function (IIEF): a multidimensional scale for assessment of erectile dysfunction. Urology. 1997;49:822-30.

20. Rosen R, Brown C, Heiman J, Leiblum S, Meston C, Shabsigh R, et al. The Female Sexual Function Index (FSFI): a multidimensional 
self-report instrument for the assessment of female sexual function. $\mathrm{J}$ Sex Marital Ther. 2000;26:191-208.

21. Konkan R, Şenormancı Ö, Güçlü O, Aydın E, Sungur MZ. Validity and Reliability Study for the Turkish Adaptation of the Generalized Anxiety Disorder-7 (GAD-7) Scale. Arch Neuropsychiatry. 2013;50:53-8.

22. Sari YE, Kokoglu B, Balcioglu H, Bilge U, Colak E, Unluoglu I. Turkish reliability of the patient health questionnaire-9. Biomed Res. 2016; Special Issue:S460-2.

23. Eskin M, Harlak H, Demirkıran F, Dereboy Ç. The adaptation of the perceived stress scale into Turkish: a reliability and validity analysis. N. Symp. 2013;51:132-40.

24. Akkus E, Kadioglu A, Esen A, Doran S, Ergen A, Anafarta K, et al. Turkish Erectile Dysfunction Prevalence Study Group, prevalence and correlates of erectile dysfunction in Turkey: a population-based study. Eur Urol. 2002;41:298-304.

25. Serefoglu EC, Atmaca AF, Dogan B, Altinova S, Akbulut Z, Balbay MD. Problems in understanding the Turkish translation of the International Index of Erectile Function. $\mathrm{J}$ Androl. 2008;29:369-73.

26. Bayraktar Z, Atun I. Despite some comprehension problems International Index of Erectile Function (IIEF) is a reliable questionnaire in erectile dysfunction. Urol Int. 2012;88:170-6.

27. Aygin D, Aslan FE. The Turkish adaptation of the Female Sexual Function Index. Turk Klinikleri J Med Sci. 2005;25:393-9.

28. Yang Y, Song Y, Lu Y, Xu Y, Liu L, Liu X. Associations between erectile dysfunction and psychological disorders (depression and anxiety): a cross-sectional study in a Chinese population. Andrologia. 2019;51:e13395.

29. Nicolosi A, Moreira ED Jr, Villa M, Glasser DB. A population study of the association between sexual function, sexual satisfaction and depressive symptoms in men. J Affect Disord. 2004;82:235-43.

30. Gillespie BJ. Correlates of sex frequency and sexual satisfaction among partnered older adults. J Sex Marital Ther. 2017;43: 403-23.

31. Palha-Fernandes E, Alves P, Lourenço M. Sexual satisfaction determinants and its relation with perfectionism: a cross-sectional study in an academic community. Sex Relatsh Ther. 2019;1-15. https://doi.org/10.1080/14681994.2019.1677884.

32. Micelli E, Cito G, Cocci A, Polloni G, Russo GI, Minervini A, et al. Desire for parenthood at the time of COVID-19 pandemic: an insight into the Italian situation. J Psychosom Obstet Gynaecol. 2020. https://doi.org/10.1080/0167482X.2020.1759545.

33. Frohlich P, Meston CM. Sexual functioning and self-reported depressive symptoms among college women. J Sex Res. 2002;39:321-5.

34. Aksaray G, Yelken B, Kaptanoğlu C, Oflu S, Özaltin M. Sexuality in women with obsessive compulsive disorder. J Sex Marital Ther. 2001;27:273-7.

35. Davis D, Shaver PR, Vernon ML. Attachment style and subjective motivations for sex. Pers Soc Psychol Bull. 2004;30:1076-90.

36. Birnie-Porter $\mathrm{C}$, Hunt $\mathrm{M}$. Does relationship status matter for sexual satisfaction? The roles of intimacy and attachment avoidance in sexual satisfaction across five types of ongoing sexual relationships. Can J Hum Sexuality 2015;24:174-83.

37. Impett EA, Peplau LA, Gable SL. Approach and avoidance sexual motives: Implications for personal and interpersonal well-being. Personal Relatsh. 2005;12:465-82.

38. Nascimento ER, Maia AC, Nardi AE, Silva AC. Sexual dysfunction in arterial hypertension women: the role of depression and anxiety. J Affect Disord. 2015;181:96-100.

39. Assari S. Intercourse avoidance among women with coronary artery disease. J Sex Med. 2014;11:1709-16.
40. Carmack Taylor CL, Basen-Engquist K, Shinn EH. Predictors of sexual functioning in ovarian cancer patients. J Clin Oncol. 2004;22:881-9.

41. Rathore C, Henning OJ, Luef G, Radhakrishnan K. Sexual dysfunction in people with epilepsy. Epilepsy Behav. 2019;100: 106495.

42. Kucukdurmaz F, Inanc Y, Inanc Y, Resim S. Sexual dysfunction and distress in premenopausal women with migraine: association with depression, anxiety and migraine-related disability. Int $\mathrm{J}$ Impot Res. 2018;30:265-71.

43. Zhou R, Li F, Chen F, Liu H, Zheng J, Lei C, et al. Viral dynamics in asymptomatic patients with COVID-19. Int $\mathrm{J}$ Infect Dis. 2020;96:288-90

44. Pan F, Xiao X, Guo J, Song Y, Li H, Patel DP, et al. No evidence of severe acute respiratory syndrome-coronavirus 2 in semen of males recovering from coronavirus disease 2019. Fertil Steril. 2020;113:1135-9.

45. Qiu L, Liu X, Xiao M, Xie J, Cao W, Liu Z, et al. SARS-CoV-2 is not detectable in the vaginal fluid of women with severe COVID19 infection. Clin Infect Dis. 2020. https://doi.org/10.1093/cid/ ciaa375.

46. Li D, Jin M, Bao P, Zhao W, Zhang S. Clinical characteristics and results of semen tests among men with coronavirus disease 2019 . JAMA Netw Open. 2020;3:e208292.

47. Brody S, Costa RM. Satisfaction (sexual, life, relationship, and mental health) is associated directly with penile-vaginal intercourse, but inversely with other sexual behavior frequencies. J Sex Med. 2009;6:1947-54.

48. Baumeister RF, Catanese KR, Vohs KD. Is there a gender difference in strength of sex drive? theoretical views, conceptual distinctions, and a review of relevant evidence. Personal Soc Psychol Rev. 2001;5:242-73.

49. Beck JG, Bozman AW, Qualtrough T. The experience of sexual desire: psychological correlates in a college sample. J Sex Res. 1991;28:443-56.

50. Balon R. Sexual dysfunction. The brain-body connection. Adv Psychosom. 2008;29:89-106.

51. Basson R. Female sexual response revisited. J Soc Obstet Gynecol. 2000;22:378-83.

52. Liu S, Han J, Xiao D, Ma C, Chen B. A report on the reproductive health of women after the massive 2008 Wenchuan earthquake. Int J Gynecol Obstet. 2010;108:161-64.

53. Kissinger P, Schmidt N, Sanders C, Liddon N. The effect of the hurricane Katrina disaster on sexual behavior and access to reproductive care for young women in New Orleans. Sex Transm Dis. 2007;34:883-6.

54. Arafat SMY, Alradie-Mohamed A, Kar SK, Sharma P, Kabir R. Does COVID-19 pandemic affect sexual behaviour? A crosssectional, cross-national online survey. Psychiatry Res. 2020;289:113050.

55. Yuksel B, Ozgor F. Effect of the COVID-19 pandemic on female sexual behavior. Int J Gynaecol Obstet. 2020;150:98-102.

56. Schiavi MC, Spina V, Zullo MA, Colagiovanni V, Luffarelli P, Rago R, et al. Love in the time of COVID-19: sexual function and quality of life analysis during the social distancing measures in a group of italian reproductive-age women. $\mathrm{J}$ Sex Med. 2020;17:1407-13.

57. Li G, Tang D, Song B, Wang C, Qunshan S, Xu C, et al. Impact of the COVID-19 pandemic on partner relationships and sexual and reproductive health: cross-sectional, online survey study. J Med Internet Res. 2020;22:e20961.

58. Li W, Li G, Xin C, Wang Y, Yang S. Challenges in the practice of sexual medicine in the time of COVID-19 in China. J Sex Med. 2020;17:1225-8. 